\title{
Marie de France et les Lais bretons.
}

(s. Ztschr. XXIX, 19).

\section{II.}

C'est naturellement dans les prologues et les épilogues entre lesquels Marie a encadré ses lais que nous irons chercher les éléments de cette seconde partie de notre étude. Parmi les prologues, il en est un qui au premier abord se distingue des autres par sa longueur insolite: c'est celui de Guigemar qui n'a pas moins de vingt-six vers. ${ }^{1}$ Il a du reste d'autres titres à notre attention. Qu'on le rapproche en effet des introductions qui précèdent les douze autres lais: on verra que c'est le seul endroit où Marie traite de matières étrangères au lai qui va suivre. Examinons en premier lieu les vers $\mathbf{I}-\mathbf{1} 8$ qui forment comme une première subdivision de ce prologue. Tout d'abord Marie s'y nomme, ce qu'elle ne fait dans aucun autre lai; puis elle s'indigne contre la bassesse de ces gens qui sont jaloux de tout succès, si mérité qu'il soit, insiste sur l'obligation morale qu'il y a de ne pas se laisser arrêter par ces critiques envieuses: et nous reconnaissons lì les thèmes qui deviendront de style et seront ressassés avec si peu de variété dans les prologues de tant de contes, fabliaux et romans. Chez elle, si ennemie du développement vague, nous sentons même ici la sincérité. 2 Mais en tout cas il est clair dès maintenaut que nous n'avons pas affaire au prélude d'un lai particulier, où n'auraient que faire ces récriminations, mais bien d̀ la

1 Le prologue de Bisclavret a I4 vers; Equitan I2; Yonec et le Chèrefeuil ı; Milun et le Chaitival 8; les Deux Amants et Laustic 6; Eliduc et Lanval 4; Fraisne 2.

2 Les prologues chez Marie ne sont pas encore ce qu'ils deviendront plus tard, dans les fabliaux par exemple, des préfaces verbeuses et vagues, où l'auteur se croit obligé de s'attarder, sans profit pour personne, avant de commencer son récit: Marie est sobre de paroles, et, si elle fait précéder le conte proprement dit d'une courte introduction, c'est toujours pour donner à ses lecteurs des renseignements précis et utiles. Dans l'une (Laustic, v. 3-6). elle nous definira un mot breton qu'elle va ensuite employer constamment dans le cours de sa narration; dans l'autre (Bisclavret, v. 3-12), avant de nous conter une histoire de loup-garou, elle nous expliquera en quelques vers ce qu'il faut entendre par ce terme insolite. Ou bien encore et surtout, elle nous dira ce qu'étaient ces fameux lais bretons et ce que sont au juste, en face d'eux, ses propres récits à elle. 
préface de la collection tout entière. Qu'on ne dise pas que d'ordinaire (Esope, Purgatoire de Saint Patrice), c'est dans l'épilogue et non dans le prologue que Marie se nomme. Dans les Lais il était tout naturel que ce fût le contraire. Qu'on y songe en effet: on ne saurait considérer les Lais comme un ouvrage ordinaire dont l'auteur embrasse dès le début le plan et le développement. Dans ses Fables, Marie se bornait à traduire un recueil déjà tout formé: on peut croire qu'elle a écrit l'Esope tout d'une haleine et l'épilogue en marque nettement la fin comme le prologue ouvre le livre. Mais dans les Lais nous avons une collection toujours ouverte, pour ainsi dire, qui pouvait se restreindre en cours de travail ou s'enrichir selon les hasards de la rencontre. Il est donc permis de croire que la composition des lais s'échelonna sur un certain nombre d'années et les indices ne manquent pas pour établir ce fait. ${ }^{1}$ Marie, très jalouse de sa gloire littéraire, ${ }^{2}$ devait donc, si elle voulait s'assurer la propriété d'un ouvrage dont elle ne pouvait dès le début prévoir la date d'achèvement, s'y nommer tout au commencement. - La deuxième partie de cette introduction (v. I 9-2 I) confirme notre hypothèse: ces trois vers nous annoncent en effet que Marie a entrepris de nous donner non un conte, mais toute une série de contes: ce sont ceux dont les Bretons ont fait les lais. Enfin les vers 22-26 forment l'introduction propre à Guigemar, et encore le vers 22

El chief de cest comencement

nous rappelle-t-il que Guigemar n'est que le premier lai de la collection, celui qui se trouve suivre immédiatement la préface générale.

S'il en est ainsi, que signifient les 56 vers qui dans le manuscrit

$1 \mathrm{Si}$ le recueil avait èté publié en une fois, un seul prologue cât suffi: Marie ne répète pas en tête de chacune de ses Fables qu'elle la doit à Alfred qui lui-même la tenait d'Esope. En fait nous trouvons les indications que Marie croit devoir donner a son public concernant l'origine des lais bretons et le rapport de ses contes avec ces lais répétés plusieurs fois dans des lais différents: c'est donc qu'ils ont été publiés sćparément et successivement. Il faut surtout citer ici, à côté du Prologue des Lais, l'introduction de Gutiscrnar et celle d'Equitan. Les successeurs et les imitateurs de Marie, étudiant le recueil de ses Lais, ne comprirent pas ce qui avait motivé tout d'abord ces répétitions: ils crurent que c'ćtait une loi du genre, et c'est ainsi que, selon toute vraisemblance, un petit prologue sur l'origine des lais en vint is être regardé comme le prélude obligé de tout lai brcton digne de cc nom. D'après M. Gröber (Grundr., II, 1, p. 597) le prologtue qu'on retrouve chez Marie et dans des lais anonymes, même avant Marie (le $C o r)$, doit par cela même avoir appartenu au conte breton. Cette remarque tombe si l'on observe que ces prologues ont précisément pour but de nous apprendre que nous avons affaire à une matière bretonne. Il est d'autre part fort douteux que le Cor soit antérieur à Marie. Voir p. 55 note I.

2 Voir Epilogue des Fables, v. I sqq.: Al finement de cest escrit ...। me numerai pur remembrance:| Marie ai num ... | Puet cel estre, cil clerc plusur | prendreient sur els mun labur: | ne vueil que nuls sur lui le die; | cil uevre mal ki sei ublie. 
Harléien précèdent les 12 lais et que $M$. Warnke place en tête de son édition sous le nom de Prologue? A les lire attentivement, on s'aperçoit vite que le nom d'Epilogue leur conviendrait mieux: ce prétendu Prologue a été en effet composé après les Lais dont il marque l'achèvement ${ }^{1}$ et c'est simplement parce qu'il renferme une dédicace qu'il a été placé en tête de la collection. ${ }^{2}$ Marie n'a songé en effet à présenter son œuvre au roi qu'assez tard: l'idée ne lui en était pas encore venue au moment de la composition de Guigemar et de Lanval: elle s'y adresse à d'autres patrons. ${ }^{3}$ Mais elle dut faire bientôt réflexion que plus le protecteur était haut placé, plus la protection serait efficace, et l'audace lui venant en même temps que le succès, elle résolut de dédier son recueil au roi. Mais peut-être fut-ce assez tard qu'elle y pensa, et en tout cas il ne semble pas qu'elle lui eût fait connaître formellement son intention avant le jour où elle lui présenta le recueil enfin complet

43 En l'onur de vus, nobles reis, ...

47 m'entremis des lais assembler par rime faire et reconter.

En mun quer pensoe et diseie, sire, ques vos presentereie.

5I Se vos les plaist a receveir, mult me ferez grant joie aveir; a tuz jurs mais en serrai liee.

$\mathrm{Ne}$ me tenez a surquidice,

55 se vos os faire icest present.

Ainsi nous tenons d'une part le point de départ de la collection, c'est l'introduction de Guigemar, d'autre part l'épilogue ou conclusion, c'est le morceau qui jusqu'ì présent a reçu des éditeurs le non de Prologue. Avant d'aller plus loin, nous devons rechercher

1 Marie y parle de ses lais comme d'une cuvre terminéc: tous les temps sont au passé. V. $41-42$, Rimi en ai e fait ditié, / soventes fois en ai veillié. Cf. v. 47, m'entremis et v. 49-50. Voyez au contraire le Prologue des Fables: A mei, ki la rime en dei faire (v. 27) et l'introduction de Guigemar v. 15 ct 21 .

2 Mall a bien vu (Ztschr.f. rom. Phil., III, p. 30I) que le Prologue a dû être écrit après les Lais et placé en tête en guise de dédicace. Mais il suppose a tort que la collection se terminait par un Epilogue où Marie se nommait et qui est aujourd'hui perdu. Dans un recueil de lais toujours ouvert, c'est dans l'introduction qu'il était naturel de se nommer, et c'est ce que Marie a fait.

3 Guigemar et Lanval sont en effet les seuls lais où Marie s'adresse directement à son auditoire. „Ö̈z, seignur, que dit Marie." Guig., v. 3 „Seignur, ne vus en merveilliez: | huem estranges, decunseilliez| mult est dolenz en altre terre, | quant il ne set u sucurs querre." Lanv., v. 35-38. [Marie ne fait-elle pas là un mélancolique retour sur sa propre situation?] Ces „seigneurs" qu'elle ne nommera plus dans aucun autre lai, ce sont naturellement les chevaliers à qui elle compte lire ses cuvres et dont elle espère ainsi gagner la protection. Il est clair qu'elle ne songe pas encore à un protecteur plus puissant, le roi. 
si l'Introduction a toujours occupé la place que lui donnent maintenant les manuscrits et les éditions: a-t-elle toujours été attachée au lai de Guigemar? ou bien n'en est-elle pas inséparable? Cela revient à se demander si Guigemar est bien le premier lai qu'ait composé Marie. Cette recherche est justifiée, comme nous le verrons tout à l'heure, par l'importance des renseignements que ce lai nous fournit. Quel est le témoignage des manuscrits sur ce point? Les vers $\mathrm{I}-\mathrm{I} 8$ qui, nous l'avons vu, forment en euxmêmes un tout complet se trouvent bien dans le manuscrit de Londres $(\mathrm{H}),{ }^{1}$ le plus important de tous, il est vrai, ${ }^{2}$ mais ni le manuscrit de Paris $(S)^{3}$ ni la traduction norwégienne $(N)^{4}$ ne les donnent. Cela signifie-t-il que ces vers n'ont été ajoutés qu'après coup par Marie, au moment où elle a complété sa collection et l'offre au roi? $\mathrm{S}$ et $\mathrm{N}$, incomplets du reste par ailleurs, ${ }^{5}$ remonteraient donc à une compilation antérieure à cette révision et qui ne s'ouvrait pas encore par cette introduction. Cette supposition ne semble guère probable. Si nous l'admettions, en effet, il faudrait conclure que Marie, sa collection complétée, a écrit au même moment non pas une introduction, mais deux: car si le Prologue de l'édition Warnke est bien réellement, comme nous l'avons démontré, un épilogue, il n'en reste pas moins que, renfermant une dédicace au roi dont Marie ne s'était avisée qu'assez tard, il devait prendre place en tête de la collection; le dernier vers (v. 56)

Ore oëz le comencement!

ne laisse pas de doute sur ce point. Croira-t-on qu'après nous avoir annoncé ainsi le commencement, Marie nous engage immédiatement ensuite dans une seconde préface qui se terminera elle aussi par l'annonce - véritable cette fois - du commencement:

22 El chief de cest comencement ... vos mosterrai ....

La bizarrerie reste, mais s'explique, si l'on admet que ces deux morceaux n'ont pas été écrits en même temps, que l'un a été composé bien avant l'autre pour servir de préface à une collection commençante et que l'autre date du moment où, la collection enfin terminée, Marie la présente au roi; elle ne s'est pas souciée alors de retoucher des vers qui étaient probablement dans toutes les mémoires, et a laissé volontairement ou à son insu, subsister

1 Ms. Harl. 978. Je conserve, pour désigner les manuscrits, les lettres adoptées par M. Warnke. Voir son édition, p. XXXVIII.

${ }^{2}$ Les vers $I-12$ sont donnés aussi par $P$ (Bibl. Nat., Fr. 2168). Cf. Warnke, Lais, p. XXXVIII.

8 Bibl. Nat., nouv. acq. franç. 1104 . Warnke, p. XXXVIII.

- Publiée par Keyser et Unger: Strengleikar eda Lioðabok, Christiania, 1850. Warnke, p. XXXIX.

$5 \mathrm{~S}$ ne donne ni Laustic, ni le Chaitizel, ni Eliduc mais en revanche donne Guingamor qui manque à $\mathrm{H}$. Eliduc ne se trouve pas dans $\mathrm{N}$, non plus que Guinganior. 
une contradiction dans les mots, trop légère du reste pour choquer aucun de ses auditeurs ou de ses lecteurs. Mais c'est pour nous un indice précieux de la vérité de notre hypothèse. Le nom de Marie qui se trouve dans ces dix-huit vers et ne se trouve que là est un autre indice aussi probant. Il serait surprenant, comme nous l'avons vu, que Marie eût attendu pour se nommer d'avoir terminé une collection dont la composition pouvait s'étendre sur plusieurs années et où chaque lai était vraisemblablement communiqué au public aussitôt qu'il était terminé; en fait elle ne se nomme pas dans le Prologue-épilogue composé sûrement après l'achèvement de son œuvre: c'est donc qu'elle devait s'être nommée ailleurs, à savoir dans la Préface de cette œuvre. Nous admettrons donc que $\mathrm{S}$ et $\mathrm{N}$ présentent dans l'introduction de Guigemar une lacune de dix-huit vers.

Peut-on expliquer cette lacune? Cela ne semble pas impossible. Le traducteur norwégien, comme l'a montré M. Meissner,' est dans une certaine mesure un adaptateur: il a notamment l'habitude curieuse de s'approprier tous les passages où Marie parle en son nom, et de les donner comme des réflexions personnelles qu'il ajoute à son original. Or il est clair que ce procédé pouvait difficilement s'appliquer aux vers $\mathbf{I}-\mathbf{1} 8$ de Guigemar. Marie y fait allusion à des circonstances très particulières qui ne convenaient guère à la situation du traducteur. On peut donc supposer que, trouvant ces dix-huit vers dans son original, il les a purement et simplement laissés de côté. ${ }^{2}$ - Que penser du silence de S? Il faut noter qu'en ce qui concerne Guigemar, S donne en général les mêmes leçons que $P$, et que $P$ reproduit, au moins en partie, les vers qui nous occupent. Il ne les comprend du reste pas: les vers $3-4$

Oëz, seignur, que dit Marie, $\mathrm{ki}$ en sun tens pas ne s'oblie

deviennent chez lui

$$
\begin{aligned}
& \text { Oëz, Seignur, que dit marit } \\
& \text { que en son tens nus ne soublit. }
\end{aligned}
$$

Les vers 13 - 18 manquent; peut-être, comme le suggère M. Warnke, ${ }^{3}$ doivent-ils leur omission à la ressemblance des derniers mots des vers 12 et 18 (mestier-mesparler); mais il est possible aussi que l'original de $\mathrm{P}$ ait été fort corrompu dans ce passage, comme

1 Die Strengleikar, Halle, 1902. Voir notamment p. 278.

2 C'est aussi l'opinion de M. Meissner, op. cit., p. 265. Noter que le traducteur norwégien ajoute rarfois d'importants développements à son texte (Meissner, p. 247-249): sûre preuve qu'il peut à l'occasion prendre des libertés avec son original. Il faut indiquer enfin que les leçons de $\mathbf{N}$ con. cordent le plus souvent avec celles de H. Voir Warnke, p. XLII et cf. Meissner, p. 200 sqq.

8 Lais, p. 5. Notes Critiques, v. 13-18. 
il l'était déjà certainement dans les vers qui précèdent. S'il en était ainsi, ne pourrait-on supposer que $\mathrm{P}$ et $\mathrm{S}$ remontent à un même original qui donnait un texte défectueux des dix-huit premiers vers de Guigemar? $\mathrm{P}$ a reproduit tant bien que mal, et sans comprendre, ce qu'il avait sous les yeux; $S$ a preféré supprimer des vers obscurs et qui ne se rattachaient nullement au reste du lai. 1

Passons maintenant à l'examen des vers 19-2I, où Marie nous fait connaître son intention de nous donner une série de contes et des vers $22-26$ où elle nous annonce le premier conte de la collection. Notons tout de suite que ces huit vers sont donnés par les trois manuscrits français et par la traduction norwégienne. Remarquons ensuite que les vers $19-21$ et $22-26$ sont étroitement liés par la rime et forment un tout inséparable. $\mathrm{Ne}$ soyons pas trop surpris de trouver ainsi dans la préface générale d'une collection l'annonce du premier morceau de cette collection: n'est-ce pas ainsi que Marie termine son prologue des Fables:

$$
\begin{aligned}
& 38 \text { Ci comencerai la premiere } \\
& \text { des fables qu'Esopes escrist, } \\
& \text { qu'a sun mestre manda e dist. }
\end{aligned}
$$

Ainsi ces deux paragraphes - l'un d'introduction générale, l'autre d'introduction spéciale ì Guigemar - ont toujours ćté soudés l'un à l'autre; de plus ils ont dû toujours précéder le lai de Guigimar: car d'une part ce lai y est clairement et spécifiquement annoncé, ${ }^{2}$ et d'autre part, s'il en était autrement, il faudrait donc admettre que le lai de Guigemar - seul de toute la collection - n'avait pas de prologue qui lui fut propre, ou que son prologue, s'il en avait un, aurait été supprimé à un moment donné pour faire place à celui que nous examinons maintenant: toutes suppositions possibles, mais peu vraisemblables.

Nous sommes ainsi amenés à conclure que le prologue du lai de Guigemar non seulement forme l'introduction réelle de la collection tout entière, mais qu'il a toujours précédé le lai de Guigemar; ou au moins cette conclusion qui n'est que très probable pour les vers $\mathrm{I}-\mathbf{I} 8$ est à peu près certaine pour les vers $\mathrm{I} 9-25$. Le lai de Guigemar est donc le premier lai composé par Marie. C'est ce qui est du reste pleinement confirmé par les manuscrits. L'ordre dans lequel les lais sont rangés dans chacune des trois grandes collections $(\mathrm{H}, \mathrm{S}, \mathrm{N})$ varie beaucoup de l'une à l'autre; mais Guigemar y est toujours placé en tête. L'unique manuscrit

1 S n'a pas l'air d'avoir été copié avec beaucoup de soin. En dehors du prologue, il offre, comparé à $H$, six lacunes de 2,4 et 6 vers: c'est ainsi qu'il ne donne pas les vers $885-6$ : que hum dit en harpe e en rote; / bone en est a oïr la note.

2 v. 22 sqq. El chief de cest comencement ... | vos mosterrai une aventure, | ki en Bretaigne la Menur | avint al tens anciënur. Cf. v. 315. 
$\mathrm{P}$ place Guigemar au second rang. Mais il faut remarquer que nous n'avons pas affaire ici d une collection: $P$ ne donne que trois lais dont l'un fort incomplètement, et plus d'un indice ${ }^{1}$ nous montre que le scribe de $\mathrm{P}$ ne soupçonnait nullement que les lais qu'il transcrivait eussent jamais appartenu ì une œuvre plus vaste.

Il semble que nous ne puissions guère aller plus loin dans cet cssai de détermination chronologique des lais de Marie. ${ }^{2}$ Mais nous tenons les grandes lignes, et cela nous suffit. Nous pouvons aborder avec plus de sûreté l'étude des différents passages par lesquels on peut se faire une idée des sources de Marie et de son originalité. Il nous faudra chercher notre premier texte non dans le Prologue, composé peut-étre assez tard, mais dans Guigemar - introduction et récit - qui étant le plus ancien des lais prend pour nous une importance singulière. Il est possible qu'une partie de l'obscurité qui couvre encore cette question des lais provienne de ce qu'on a mis toute sorte de textes pêle mêle, sur le même plan. Disposés suivant un ordre chronologique, peut-être vont-ils s'éclairer d'une lumière nouvelle.

Or que nous dit Marie dans Guigemar au sujet des lais?

Les contes que jo sai verais,

20 dunt li Bretun unt fait les lais, vos conterai assez briefment.

El chief de cest comencement sulunc la letre e l'escriture

24 vos mosterrai une aventure, ki en Bretaigne la Menur avint al tens anciënur.

1 C'est ainsi que le v. 19 de Guigemar "Les contes que jo sai verais" devient "Li contes que sai est verais". [Le ms. C qui ne contient que Lanval transforme semblablement le premier vers de ce lai "L'aventure d'un altre lai ... | vus cunterai" en "L'aventure de un lay ..."] Voir encore Yonec, v. 558-562 et la note critique de M. Warnke, p. 145 .

2 Les prologues nous font sentir une progression chronologique, sans nous permettre d'en déterminer exactement la loi. Equitan a da suivre Guigemar d'assez près: les mêmes idćes sont reprises dans le prologue, mais plus précises cette fois et plus détaillécs. Dans Guigemar il était simplement question des "contes, dunt li Bretun unt fait les lais" (v. 19-20). Dans Equitan, nous apprenons que ces Brctons étaient ceux d'autrefois (v. 3 ), que c'étaient les barons de Bretagne eux-mêmes (v. I-3) et qu'ils faisaient les lais pour conserver le souvenir de certaines aventures qui étaient arrivées à eux ou à d'autres (v. 3-8). Ailleurs l'introduction est très brève et presque incomplète par elle-même; c'est sans doute que le lai en question devait être joint à un autre: ainsi pour le lai de Lanval. (Cf. v. I, L'aventure d'un altre lai ... vus cunterai.) Peu à peu le public se familiarisa avec la tentative et le nom même de Marie; la poétesse n'eut plus à renouveler ses explications: il lui suffit d'annoncer que c'était un lai qu'elle présentait à ses auditeurs, et l'on comprenait (Le Fraisne, v. 1-2). Parfois elle fait allusion à la collection qu'elle a entreprise, au recueil qui est en voie de formation (Bisclavret, v. I -2; Yonec, v. I-4; Milun, v. 1-4). C'est une façon de signer le lai, de rappeler que d'autres l'ont précédé, que d'autres le suivront. 
Et dans l'épilogue revenant sur le même point et le précisant:

De cest cunte qu'oï avez

884 fu Guigemar li lais trovez, que hum dit ${ }^{1}$ en harpe e en rote;

bone en est a oïr la note.

Les vers sont limpides et le sens en est net. Notons tout de suite le soin avec lequel Marie s'applique à distinguer entre le lai breton de Guigemar - composition musicale chantée - et l'histoire du héros telle qu'elle nous la rapporte dans sa narration poétiquc. Il est à remarquer qu'elle n'emploie pas une fois le mot lai pour désigner son conte: elle réserve rigoureusement ce terme pour la composition bretonne. Quant à elle, elle se contente de nous 'montrer une aventure' et de nous 'conter un conte': le premier terme se référant à la matière, le second à la forme du poème. On a fait cette remarque plus d'une fois, ${ }^{2}$ mais sans en tirer, semble$\mathrm{t}$-il, les conséquences qu'elle comporte. Rappelons-nous que Guigemar est le premier en date de ses lais, que c'est là qu'elle doit expliquer son innovation, si elle innove, en tout cas faire connaître au lecteur le plan général de l'ouvrage qu'elle entreprend; faisons attention qu'en fait c'est là qu'elle nous a donné les renseignements les plus significatifs, puisqu'en dehors du Chevrefenil le lai de Guigemar est le seul à propos duquel elle mentionne nettement une mélodie, et demandons-nous s'il n'est pas étrange qu'elle persiste d̀ appeler son poème un conte, à nous dire qu'elle va nous conter des contes, bref à ne pas plus mentionner le mot de lai au sens de poème narratif que si elle n'avait jamais entendu parler d'une telle signification. A cela il n'y a qu'une explication possible: c'est qu'au moment où Marie écrit Guigemar, elle ne connaît en effet pas ce sens. Pour elle, un lai c'est une composition que l'on dit en harpe et en rote: et pour son public il en est de même. De là ces simples mots qui étaient clairs pour tous: „Les contes ... | dunt li Bretun unt fait les lais | vos conterai." Les

1 Dit est la leçon de P. Mais M. Warnke établit dans son introduction (p. XLIII) que pour le lai de Guigemar c'est $\mathrm{H}$ qui doit être préféré à tous les autres manuscrits. Or $\mathrm{H}$ porte non dit, mais fait (que hum fait en harpe et en rote). [Les vers $885-6$ manquent dans le 3 ème ms. S]. Il faut conscrver ici comme ailleurs le texte de H. En dehors des cas nombreux oì "faire un lai" signifie composer un lai, il y a quelques exemples assez nets, semble-t-il, où la même locution signifie exécuter un lai (soit sur la harpe, soit en le chantant). Voir le passage si souvent cité du Tristan de Thomas, où il nous montre Iseut qui „en sa chambre se set un jur | e fait un lai pitus d'amur ..." (Ed. Bédier, v. 833-834). Cf. Richeut (Méon, Nouv. rec., I, p. 63): „Plus set Sansons | Rotruange, conduiz et sons: | Bien set faire les lais bretons." Horn, Ed. Brede et Stengel, v. 2777 et 2779. Peut-être avons nous le même emploi dans un passage assez obscur du lai du Cor: "Seingnours, cest lai troua | Garaduc qui fest la" (Ed. Michel, dans Wolf, Ueber die Lais. p. 327 sqq., v. 583-584), c'est à dire: „Scigneurs, l'auteur de ce lai est Garaduc, celui même qui vient de l'exécuter devant vous". p. XXVI.

2 Voir notamment G. Paris, Rom., XIV, p. 606 et Warnke, Lais 
auditeurs entendaient par là que Marie allait leur dire un conte qui, sous une forme antérieure, était à l'origine d'un de ces lais bretons, dits en harpe et en rote, que tous connaissaient si bien. Mais que dès lors Marie et son public appelassent un conte qui se présentait dans ces conditions un lai, c'est ce que rien ne nous permet d'affirmer. Le texte de Marie nous permet même d'affirmer précisément le contraire.

Faute d'avoir vu cela, on est tombé dans des complications dont il ne semble pas qu'on soit encore sorti. Il faut aller chercher assez loin l'origine de cette erreur, puisqu'elle remonte jusqu'aux premiers critiques qui se soient occupés de ces questions, de la Rue, Roquefort, Raynouard, Ellis, Warton, Ritson. Selon ces savants le nom de lai, à s'en rapporter à l'usage des trouvères anglo-normans et de leurs plus anciens imitateurs français et anglais, s'appliquait à l'origine seulement à des poèmes narratifs et en particulier à ceux qui étaient chantés ou récités sur des mélodies bretonnes ou qui reproduissaient de vieilles traditions et chansons populaires bretonnes. ${ }^{1}$ Ainsi ces premiers lais - lais de chevalerie, lais historiques, comme on les appelait encore - se distinguaient nettement, dans l'ordre chronologique, des lais postérieurs lyriques. F. Wolf, dans son beau livre Ueber die Lais, Sequenzen und Leiche, paru en 1841, réfuta victoricusement cette thèse. Par des exemples nombreux et probants, il montra que de Wace (I I55) aux trouvères du XIII ${ }^{e}$ siècle, - moment où les lais lyriques apparaissent à côté des lais épiques - le mot de lai se dit non seulement des lais épiques, mais se prend dans le sens général de chanson, mélodie, chant, air. Après le livre de Wolf il resta prouvé que lai au sens de composition musicale quelconque était fort ancien dans la langue; mais de la thèse opposée, désormais réfutée dans ses prétentions extrêmes, il se conserva néanmoins l'opinion que lai au sens de poème narratif n'était pas moins ancien. En réalité, il faut aller, dans la même voie que Wolf, plus loin que lui. Non seulement lai signifiant chanson n'est pas postérieur dans le temps à lai signifiant conte, mais il lui cst antérieur: nous avons là le sens ancien et original du mot. L'autre n'est qu'un dérivé dont nous aurons d̀ établir la date d'apparition. Je ne connais en effet pas de textes antérieurs à Marie où on trouve le mot lai dans ce sens. Qu'on parcoure les nombreux témoignages réunis par Wolf, $P$. Paris et F. Michel: ${ }^{2}$ partout, à cette première époque, lai signifie mélodie, chanson ou quelque chose d'analogue. Il faut aller, en p. $2-3$.

1 Je résume dans tout ce passage une page de Wolf, Ueber die Lais,

2 Il est évident que dans l'intérêt même de sa thèse Wolf devait avant tout réunir des exemples de lai au sens de chanson. Il reste néanmoins curieux que, dans cet amas d'exemples accumulés par lui, il ne se soit pas glissé de cas de l'autre sens (exception faite naturellement de Marie et de ses imitateurs). - P. Paris, Rom. de la Table Ronde, 1, p. 6-24; F. Michel, Tristan, T. I-1 1, 1835 . 
dehors de Marie, bien près du XIII ${ }^{e}$ siècle pour trouver l'autre sens nettement attesté, et même alors l'emploi en est singulièrement limité. Chrétien mentionne des lais à plusieurs reprises, mais ce ne sont jamais des lais épiques; ${ }^{1}$ chez Wace et postérieurement chez Thomas, chez Gottfried de Strafsburg qui traduit Thomas, il n'est question que de lais lyriques, chantés en harpe et en rote, jamais de lais narratifs. Le fabliau de Richeut, le roman de Troie, la chanson de Horn, la Chronique de l'abbaye du Mont St. Michel de Guillaume de St. Pair, Parténopeus de Blois, la Geste des Loherains, le roman des Sept Sages, ${ }^{2}$ pour ne nous en tenir qu'à des textes relativement anciens, connaissent bien les lais, mais ils n'entendent par là que des airs de musique ou des chansons. D'autre part nous avons de nombreuses allusions à ces conteurs ambulants qui s'en vont de cour en cour, de château en château, colportant anecdotes et historiettes: 3 il est curieux que pas une fois, à ma connaissance, on ne leur fasse "conter un lai ". Cil autre jongleur chantent et dient lais, lisons-nous dans Gautier d'Aupais, Mis je sur .i. conteres qui leur matere lais. 4 De quelque côté que nous nous tournions, ${ }^{5}$ la même conclusion semble s'imposer: le lai ne se sépare pas de la vielle ou de la harpe; qui dit lai dit chant.

1 Cf. Foerster, grande éd. d'Erec, Halle, 1890, p. 297.

2 Quelques uns de ces livres me sont restés inaccessibles. Je les mentionne d'après les citations qu'en font Wolf, P. Par is ou Michel.

8 Voir en particulier les allusions recueillies par G. Paris dans Hist. litt. de la France, t. XXX, Romans en vers du Cycle de la Table Ronde, Introduction générale.

- Cité par Wolf, op. cit., p. 157, n. 5 .

- Seul un passage d'Ille et Galeron (Ed. Foerster, v. 929-930) semble offrir, à cette première époque, l'autre sens. (Voir la note de M. Foerster, p. 188: „damit können hier nur die kleineren, damals sehr in Schwung betindlichen erzählenden Gedichte gemeint sein.") A la rigueur, le roman étant de 1167 , on pourrait soutenir, et l'on a soutenu, qu'il y a là une allusion à un lai de Marie, Eliduc, qui serait la source du roman. Mais pour d'autres motifs encore le passage est a écarter. Il est nécessaire de le citer tout au long pour rendre la démonstration claire: „Car en amor a maint degré: $\mid \mathrm{Al}$ commencier est de bon'aire, | Le gent blandist por mix atrairc; | Et puis, quant il est ore et leus, | Reset bien mostrer de ses jeus. / Cist n'en ont pas encor grant cure, | Bien lor ira, s'issint lor dure; | Mes s'autrement n'alast l'amors, | Li lais ne fust pas si en cours, | Nel prisaissent tot li baron. | Grant cose est d'Ille et Galeron:| N'i a fantome ne alonge, | $\mathrm{Ne}$ ja n'i troverés mençonge. | Tex lais $i a$, qui les entent, | Se li sanlent tot ensement | Con s'ëust dormi et songié" (v. $92 \mathrm{I}-936$ ). Que signifie le vers 929 ( $\mathrm{Li}$ lais ne fust pas si en cours)? De quel lai s'agit-il ici? Evidemment de celui qui est mentionné deux vers plus bas, v. 931: „Grant cose est d'Ille et Galeron." Mais par là ne peut-être désigné que le roman même d'Ille et Galeron. Cf. v. 932 -933: „N'i a fantome ne alonge, | ne j'a n'i troverés mençonge." Ce futur semble bien indiquer qu'il s'agit de l'ouvrage même que l'on a sous les yeux ou que l'on entend lire. Est-ce que les vers 934-936 n'instituent pas une comparaison entre Ille et Galeron qui ne rapporte que des choses véridiques et tels (autres) lais qui se fondent sur de pures imaginations? Je ne puis croire en effet avec M. Foerster (introd. p. XXIV, note 34) qu'il s'agisse clans les vers 934-936 de manvais lais ennuycux (schlechte weil langweilige Lais) et j'interprète ainsi tout le passage: l'amour n'est pas toujours aussi uni que ses 
Il faut conclure qu'antérieurement à Marie lai ne signifie que chanson ou mélodie; et comme c'est chez elle que nous trourons le premier emploi du mot au sens de conte, narration, il semble assez naturel de lui en attribuer l'introduction dans la langue. D'autre part nous avons vu qu'au moment de la composition de son premier conte Guigemar elle ne connaissait pas ce sens nouveau d'un vieux mot. Elle n'a donc dû s'en aviser que peu à peu: voyons comment elle y a été amenée. Guigemar n'est pas à beaucoup près le seul de ses poèmes qu'elle évite d'appeler lai: Lanval, les deux Amants, Laustic, Eliduc, Guingamor sont dans le même cas. Il faut joindre à cette liste Equitan et Chaitivel, quoique les vers $9-10$ du premier et $\mathrm{I}-2$ du second puissent à première vue sembler équivoques. Il y a donc huit lais - sur treize où Marie applique systématiquement les mots 'aventure' et 'conte' à son poème, réservant le terme lai pour la composition bretonne (de quelque nature que fût celle-ci). Ceci est assez significatif. Cependant ceétait bien un genre nouveau que Marie créait là: elle en avait conscience. Voyez le Prologue, v. 28 sqq.: elle abandonne son projet de traduire du latin une "bonne estoire“ parce que

commencements peuvent le faire supposer: c'est ce que témcigne un lai (entendez Ille et Galeron) qui doit rapporter des choses véritables puisqu'il a recontré telle faveur, bien différent en cela de ces lais qui nous racontent des histoires merveilleuses, inventées à plaisir, semblables aux créations des rêves. [Le lai d'Eliduc avec sa morte qui ressuscite rentrerait assez bien dans cette dernière catégorie.] - D'autre part, nulle part ailleurs Gautier ne mentionne que son roman est fondé sur un lai: il appelle son poème trovëure au v. 6 et sa source esture au v. 6590 , et il n'y a pas d'autre indication. - De tout cela on est fondé à conclure que c'est bien son roman d'Ille et Galeron qu'il entendait désigner sous le nom de lai au v. 929 et que c'est encore ce roman qu'il oppose au v. 934 à d'autres lais. Il reste une difficulté: comment peut-il appliquer a son roman le vers 929 , ne fust pas si en cours" avant de l'avoir fait connaître? Peut-être faut-il voir dans tout le passage une addition postéricure à la composition du roman: cette addition peut être soit le fait de Gautier lui-même, après le succès de son auvre, soit d'un jongleur qui après lui „exploitait" le poème. Ce qui rend cela plus vraissemblable encore, c'est que tout le morceau a l'air d'une réminiscence d'un passage analogue de Denis Pyramus. M. Foerster a signale l'analogie, p. XXII (naturellement pour lui s'il y a eu un imitateur, ce serait Pyramus.) Mais elle est plus complète qu'il ne l'indique. Comme Gautier, Pyramus se vante de ne nous rapporter dans sa Vie de Saint Edmund que des choses véritables, "ke sun de sen e si verray | K'unkes rien ne pout plus veir estre; ... | Ceo que homme veit, ceo deit hom crere; | Kar ce n'est pas sunge ne arueire" (v. $70-71,77-78)$. Un peu plus haut il reproche a Marie de composer des vers , ke ne sunt pas de tut verais" et à l'auteur de Partonope la 'vanité' des ses récits: „Cum de fable et de menceonge / La matire resemble suonge, / Kar ceo ne put unkes estre" (v. $3 \mathrm{I}-33$ ). [Je cite d'après Fr. Michel, Rapports au ministre, p. 258 sqq. Collect. de docum. inéd. sur l'hist. de France.] Ainsi, que nous référions le v. 929 d'Ille et Galeron à Eliduc ou que nous voyions dans tout le passage (v. $92 \mathrm{I}-936)$ une addition postérieure à la composition du roman, - ce que je crois plus vraisemblable - des deux côtés nous revenons a Marie et le passage perd toute valeur quand il s'agit de déterminer ce qui existait avant Marie. [Voir une note de G. Paris, Rom., XXI, p. 278, n. I, qui m’avait échappé au moment où je rédigeais cet article.] 
tant d'autres l'ont fait déjà. Et les vers suivants ${ }^{1}$ nohs donnent bien l'impression qu'elle croit avoir trouvé finalement quelque chose de neuf, d'original. Mais à un genre nouveau il faut un nom: 'aventure' ne désigne que la matière, la fable du conte, et le terme 'conte' lui-même est trop peu spécifique. Or il y avait des chances pour que le mot lai, qui revenait si souvent dans ses prologues et épilogues et qui appartenait à la langue de la technique musicale mais pas encore à celle de la technique littéraire, fût le mot qu'elle finirait par choisir, sans peut-être l'avoir spécialement voulu, par la force des choses. On saisit bien la transition dans des passages où elle hésite encore. Comparez le début du Chivrefeuil "Asez me plest ... del lai ... que la vérité vus en cunt"“ avec la fin "Dit vus en ai la vérité, | del lai que j'ai ici cunté." $\mathrm{Ce}$ sont les mêmes mots, mais l'ordre est renversé. Comparez encore le début de Milun: „Ici comencerai Milun | e musterrai... pur quei e coment fu trovez / li lais hi issi est numez" avec la fin "firent un lai li anciën; | e jeo ki l'ai mis en escrit ..." Dans Yonec le passage d'un sens à l'autre est presque achevé:

I Puis que des lais ai comencié,

ja n'iert pur nul travail laissié;

les aventures que jeo sai,

tut par rime les cunterai.

Puis nous avons des textes qui deviement plus nets encore: „Le lai del Fraisne vus dirai | sulunc le cunte que jeo sai." Fraisne, v. I-2. (Et là encore le mot sulunc nous atteste le passage d'un sens à l'autre.) „Quant des lais faire m'entremet | ne vueil ublier Bisclavret." Biscl., v. I-2. (Cf. la fin: "L'aventure qu'avez oïe) veraie fu ... De Bisclavret fu fez li lais.") Cest ainsi que peu à peu, pour la commodité de l'expression, pour éviter dans un court prologue de longues périphrases, Marie a été conduite à employer le mot lai dans un sens qu'il n'avait pas encore eu en français et dont elle ne s'était avisée elle-même qu'assez tard. Elle y fut vraisemblablement aidée par le public qui dut trouver le mot commode pour désigner une chose nouvelle. Il est curieux cependant de constater qu'un contemporain de Marie, Denis Pyramus, ayant occasion de mentionner les contes de la poètesse les appelle tout d'abord non des lais tout court mais des vers de lais. ${ }^{2}$ Là encore nous voyons cette même fluctuation de sens que nous signalions tout à l'heure chez Marie. Arrivons maintenant au Prologue qui, ne l'oublions pas, est un épilogue et une conclusion. Voici ce que Marie nous y dit sur le sujet qui nous intérese: „ayant renoncé à traduire du latin, j’ai songé aux lais que j'avais

1 „mais ne me fust guaires de pris: | itant s'en sunt altre entremis. | Des lais pensai qu'oïz aveie" (v. $3 \mathbf{I}-33$ ).

2 ,E dame Marie autresi | $\mathrm{ki}$ en ryme fist e basti | e composa les vers de lays". Un peu plus loin, le simple lai va, pour la commodité, remplacer cette périphrase mal commode: "Les lays suelent as dames plaire ..." 
entendus. Je savais fort bien que ces airs de musique avaient été composés pour conserver le souvenir de quelque aventure: or j'ai entendu raconter plusieurs de ces aventures que je ne veux pas laisser tomber en oubli. J'ai passé beaucoup de nuits à les mettre en rime." Ceci me semble l'interprétation la plus rigoureuse et la plus nette du passage. On pourrait, il est vrai, rapporter le en du vers $39^{1}$ non à aventures du vers 36 mais à lais du vers 33 . On l'a fait ${ }^{2}$ et il est possible que ce soit la vraie façon d'interpréter le passage. Au fond cela nous importe peu, car il n'est pas douteux qu'au moment où elle écrivait ces vers Marie n'eût déjà été amenée à donner au mot lai le sens nouveau que nous avons signalé. Cependant elle n'avait indiqué cela nulle part expressément. Présentant sa collection de contes au roi elle lui devait et elle devait au public une définition du néologisme qu'elle avait introduit dans la langue. Or cette définition elle la donne, à mon sens, dans les vers suivants du Prologue:

43 En l'onur de vus, nobles reis, ...

47 m'entremis des lais assembler

par rime faire e reconter.

Le par du v. 48 introduit une explication du vers précédent. "Je travaillai d rassembler des lais - non pas (comme on pourrait le croire en s'en tenant au sens strict du mot) en recueillant des mélodies ou des chansons - mais en versifiant des contes."

Un point est acquis: au moment où Marie commence d̀ rassembler et à écrire ses contes, on ne connaît, en France et en Angleterre, en fait de lais que des lais bretons, que suivant le texte de Guigemar on dit en harpe et en rote. Il est intéressant maintenant de rechercher quelle est la nature du rapport qui unit ces lais bretons aux contes de Marie. Assurément les seconds ne viennent pas des premiers: car Marie nous dit précisément le contraire: "De cest cunte qu'oï avez | fu Guigemars li lais trovez." Le poème français ne vient donc pas du lai breton, mais le lai breton vient d'une version, plus ancienne naturellement, du conte français. Qu'est-ce au juste que ce lai breton? Certainement une mélodie et très probablement aussi des paroles sur lesquelles se chantait la mélodie. L'air en est agréable à entendre, nous dit Marie: elle l'a donc entendu. Confidence précieuse qu'elle ne nous renouvellera pas. Une fois encore, dans le Chevrefeuil elle fera allusion à ce sens musical du mot lai: „Tristan qui bien saveit harper | En aveit fait un nouvel lai." Sans nous dire du reste qu'elle ait entendu cet air 'nouvel'. Et puis c'est tout: dans les

1 "Plusurs en ai oïz conter". - Si l'on accepte l'interprétation que je propose (après d'autres), le genre masculin de ö̈z ne doit pas arrêter. Cf. Elid., v. 25-26: kar des dames est avenu | l'aventure dunt li lais fu.

\& Warnke, Lais, p. 226 (note au v. 39 du Prologue) rapporte l'opinion de M. Ahlström, qui est celle que j'ai reprise pour mon compte, mais ne parait pas disposé à l'accepter.

Zeitschr. f. rom. Phil. XXIX. 
onze autres contes il est encore question de lais, la plupart du temps bretons, mais elle n'ajoute jamais la plus légère indication qui puisse nous faire supposer qu'elle pense à une mélodie ou à une chanson.' Chose singulière, si à la façon dont elle nomme 'Guigemar li lais' on a l'impression qu'il s'agit là d'une composition connue, cette impression est singulièrement différente dès que nous passons aux autres lais. La plupart n'ont pas l'air d'être connus: elle a l'air d'en apprendre les noms à ses auditeurs. Voyez le début de Laustic: "Une aventure vus dirai| dunt li Bretun firent un lai. | Laüstic a nun, ceo m'est vis, | si l'apelent en lur païs." Voyez le Chailivel: "Talenz me prist de remembrer | un lai dunt jo ö parler. | L'aventure vus en dirai | e la cité vus numerai | u il fu nez, et cum ot nun." Ces deux passages sont les plus significatifs, mais les autres laissent la même impression: en plus d'un cas Marie apprend à ses auditeurs non seulement l'origine d'un lai, mais le nom même de ce lai. L'idée que se fait ou semble se faire Marie de ces lais bretons est du reste curieuse. Examinez les épilogues de la plupart des contes; il nous y est toujours dit, sous une forme ou sous l'autre, que le lai a été composé pour conserver le souvenir de telle ou telle aventure. A s'en tenir strictement aux paroles de Marie, le lai semble être, pour elle, moins une composition musicale ayant une valeur esthétique propre qu'un moyen mnémotechnique ingénieux de se rappeler telle action héroïque, tel ou tel personnage fameux. Le Bisclavret rappelle l'histoire de ce loup-garou qui fut trompé par sa femme. Equitan rappelle l'histoire de ce roi qui voulant faire périr son sénéchal dont il avait séduit la femme fut pris à sa propre ruse. Mais comment le lai conservait-il le souvenir de l'aventure qui lui avait donné naissance? Il est impossible de ne pas répondre que c'est par le nom qu'il portait. Et c'est bien en effet sur la dénomination du lai que Marie insiste: le nom devait être typique, rappeler soit le héros principal soit une circonstance. caractéristique de l'aventure. De là ces discussions sur le véritable titre que doit porter tel ou tel lai.2 On disait autrefois le lai d'Eliduc, on dit maintenunt le lai de Guildeluec et Guilliadun, „car des dames est avenu / l'aventure dunt li lais fu." Doit-on dire le Chaitivel ou les Quatre deuils? c'est une question que les auteurs du lai ainsi nommé se sont posée et qu'ils n'ont résolue qu'après

1 Il n'est peut-être pas inutile de rappeler que nous avons écarté, comme sans valeur, les renseignements fournis par Graelent et Doon, qui concurremment au texte de Guigemar ont fourni la base de plus d'un système. Ce dernier seul doit être retenu. De là son importance.

2 C'est là un trait qui n'a pas manqué de frapper les imitateurs de Marie et qu'ils ont reproduit à l'envi. Voir l'Espine, v. 507-512, le Lecheor, v. I19-122, et aussi Ignaure (Bartsch, p. 568, v. 37 sqq.): dans ce dernier cas, il est vrai, on peut soupçonner que 2 poèmes différents et d'étendue fort inégale, œuvres du reste du même poète, ont été soudés ensemble. Ignaure est un fabliau spirituel, et le Lay del Prison, un petit poème miève et précieux dans le genre de l'Ombre ou du Conseil, quoique plus court. 
une discussion approfondie: c'était donc, semble-t-il l'important pour eux, c'est à dire au fond pour Marie. ${ }^{1}$ Dans tout cela rien qui nous fasse songer au mérite musical, à l'importance esthétique de ces lais. La seule question est de savoir s'ils sont bien ou mal, heureusement ou maladroitement nommés. C'est là une conception assez etrange du lai, et il est difficile de croire que telle ait bien été au fond l'opinion de Marie. Mais elle nous prouve simplement que tout ce que Marie connaissait de ces lais, c'en était le nom, - ou pour mieux dire c'en était l'aventure' .qui était à un moment donné censée leur avoir donné naissance. Et ces aventures qu'elle nous conte, elles lui sont arrivées par ailleurs, nullement liées à des lais bretons: le texte souvent cité déjà de Guigemar en fait foi. Il n'est donc pas très exact de se représenter les lais de Marie comme les livrets de mélodies bretonnes connues. ${ }^{2}$ Son point de départ est au contraire dans l'aventure' qu'elle nous raconte pour elle-même, parce qu'elle en a senti le charme, nullement pour illustrer une prétendue mélodie bretonne dont il reste dans la plupart des cas à démontrer l'existence.

Il est cependant vrai qu'elle ne manque jamais d'ajouter que les Bretons ou les anciens „en firent un lai", et ceci même est un fait qu'il nous faut expliquer. Pourquoi recommander des aventures qui trouvent en elles-mêmes leur intérêt et leur justification du nom des lais bretons? Simplement parce que les lais bretons étaient alors fort à la mode. Marie, qui cherchait volontiers des voies nouvelles, ${ }^{3}$ a trouvé là un moyen ingénieux de donner du piquant à ses contes: elle les faisait bénéficier de l'intérêt qu'on portait alors à tout ce qui touchait à la musique des Celtes. Cette musique était très renommée en France et en Angleterre: Hertz 4 en cite dans son Spielmannsbuch des témoignages curieux. De son côté Wolfs avait déjà réuni plus d'un texte "qui prouvait le plaisir qu'on prenait soit au Nord soit au Midi de la France d ces mélodies bretonnes." Ces mélodies furent-elles jamais chantées - en dehors des pays celtiques - sur des paroles bretonnes? C'est possible, mais aucun témoignage ne le prouve. En tout cas

1 Voyez comme l'auteur du lai de la Plage (conservé seulement dans la traduction norwégienne) se représente la composition de cette chanson dont il raconte l'origine. Guillaume le Conquérant, voulant perpétuer le souvenir d'une belle journée passée au bord de la mer à Barfleur, demande à la ,femme rouge" de Bretagne, qui connaissait a fond l'art de la harpe, de lui composer une nouvelle chanson sur la plus belle mélodie possible: mais le nom de la chanson est déjà trouvé, elle s'appellera la chanson de la plage; il ne reste plus qu'à composer la musique et les paroles. N'est-ce pas significatif? [Je me suis servi de la traduction allemande de la version norwégienne, donnée par Hertz dans son Spielmannsbuch ${ }^{2}$, p. 53-54.]

2 Idée exposée et souvent reprise par G. Paris (Voir en particulier Rom., VII, p. I; VIII, p. 33; Hist. Litt. de la France, t. XXX, p. 7-8) et qui est devenue courante.

8 Voir Prologue, v. 31-32.

- Spielmannsbuch ${ }^{2}$, p. 44-46.

- Ueber die Lais, p. 7 . 
c'est l'air qui charmait et non les paroles. On dut même bientôt adapter des paroles francaises à ces mélodies celtiques; on dut même continuer à appeler lais bretons des mélodies composées par des Français à l'imitation des airs spécialement celtiques. Qu'était-ce au juste que ce lai de Guigemar, que Marie nous dit avoir entendu? Une mélodie proprement bretonne, semble-t-il, à en juger par le nom qu'il portait, et probablement exécutée en harpe ou en rote par un Breton. Y avait-il des paroles? S'il y en avait, étaient-elles bretonnes? Impossible de répondre avec certitude d̀ ces questions. Pourtant si les paroles eussent été bretonnes, il semble curieux que Marie n'eût pas fait la moindre allusion à une circonstance au fond étrange. Quoi qu'il en soit elle a été frappée par ce nom de Guigemar qui lui a rappelé un conte lu ou entendu quelque part. Ou encore la mention de ce nom dans un conte qu'elle avait sous les yeux ou entendait raconter lui a rappelé un lai de Guigemar qu'elle avait jadis entendu chanter ou exécuter. Voilà le point de départ de sa collection: elle va nous dire une aventure qui est précisément celle à propos de laquelle les Bretons ont fait le lai de Guigemar, et en chercher d'autres qui expliquent également l'origine d'autres lais célèbres. Ces aventures sont du reste authentiques: elles sont bien arrivées, il y a très longtemps, dans la mystérieuse Bretagne, telles que Marie nous les rapporte:

\section{Les contes que jo sai verais, 1 dunt li Bretun unt fait les lais, vos conterai.}

Il y avait là vraiment de quoi piquer la curiosité du public. Naturellement, partant non du lai, mais de l'aventure, Marie ne trouvait pas toujours un lai, c'est à dire une composition musicale connue, qui y correspondît. Mais même dans ces cas elle devait mentionner quand même que les Bretons en firent un lai, ce qui n'était après tout qu'une façon piquante de marquer l'origine celtique $\mathrm{du}$ conte. Cette origine pouvait du reste être en certains cas assez douteuse: il y a telles de ses histoires qui non seulement n'ont rien de proprement celtique, mais qui très vraisemblablement ne proviennent pas d'une source celtique. Dans ce nombre il faudrait ranger Fraisne et les deux Amants. Cette dernière légende a fort bien pu être recueillie par elle-même en Normandie, et il semble bien que dans les vers 250-25 I „Pur l'aventure des enfanz| a nun li munz des Dous Amanz" nous ayons la conclusion du lai exprimée dans une des formules qui lui sont ordinaires. C'est par une sorte d'habitude mécanique ou par un désir de symétrie qu'elle ajoute les deux autres vers „Issi avint cum dit vus ai. $/ \mathrm{Li}$

1 Denis Pyramus ne partage pas la confiance de Marie: Dame Marie composa "les vers de lays |ke ne sunt pas du tut verais." Il lui emprunte ses rimes pour lui donner un démenti. 
Bretun en firent un lai" $(25 \mathrm{I}-2)$ qui font presque double emploi avec les deux précédents. Remarquons de plus qu'd plusieurs reprises elle ne mentionne pas les Bretons comme auteurs du lai (le Fraisne, Yonec, Milun, Chaitivel, Chievrefueil), et qu'enfin dans le Prologue-Epilogue il n'est pas question d'elix: les Bretons ont fait place à "ceux qui composèrent ces lais." N'est-ce pas dire expressément, la collection une fois terminée, que tous les lais n'en sont pas également d'origine celtique?

Nous sommes dès maintenant en mesure de répondre avec précision à deux questions difficiles, dont on a proposé bien des solutions, parfois très ingénieuses, mais rarement convaincantes. ${ }^{1}$ I 0 . Quel rapport y a-t-il entre les lais narratifs et les lais lyriques français? Pourquoi le même mot pour désigner des genres si différents? $2^{0}$. Quel rapport y a-t-il entre les lais de Marie et les lais bretons auxquels elle se réfère sans cesse? Comment passer de chansons de harpes d̀ des contes faits pour la lecture?

I0. La difficulté est en voie de disparaître si l'on remarque que des deux sens en question, l'un seul est ancien, appartient à l'usage courant, est universellement accepté par tous ceux qui parlent français, tandis que l'autre est un néologisme dû̀ à la fantaisie plus ou moins consciente d'un écrivain déterminé et ne s'est jamais fait accepter au delà d'un cercle très limité. Le mot lai, on s'accorde à le reconnaître aujourd'hui, vient du celtique 2 Il correspond à l'irlandais laid, qui signifie chanson et chant des oiseaux. Ce dernier sens montre que ce qui domine dans le mot c'est plus tôt encore l'idée d'air que de chanson, de mélodie que de paroles susceptibles de s'adapter à une mélodie. C'est bien ainsi que nous trouvons le mot employé en français dès les premiers exemples: il désigne souvent ici aussi le chant des oiseaux. Mais i côté de cette signification générale le mot a dû avoir de très bonne heure - et précisćment en vertu de son origine - le sens plus restreint de mélodie d'une nature particulière: il s'est

1 Pour la rère question, voir Wolf, Ueber die Lais; Birch-Hirschfeld, dans Ersch und Grubers Alig. Encyklopädie, s. v. lais. Cf. Warnke, Lais, p. XXI-XXIII. Pour la 2 ème question, voir G. Paris, articles cités page 307, note 2; Brugger, Zls. f. fr. Spr. u. Lit., XX, p. 134, note 74; Gröber, Grundr., II, I, p. 591; Suchier, Gesch. der fr. Lit., p. 119; Warnke, Lais, p. XXIV sqq.; Bédier, Rev. des deux mondes, I89I, p. 849 . Selon M. Bédier, les lais des jongleurs étaient mi-parlés, mi-chantés. Les jongleurs interrompaient leur récit pour chanter sur la harpe certaines parties de la légende plus propres à revêtir une forme lyrique. (Cf. Aucassin et Nicolette.) Cette hypothèse est la plus séduisante de toutes. Mais il reste que rien ne nous autorise à voir un lien quelconque entre ces "chantefables" et les lais de Marie.

2 Voir Thurneysen, Keltoromanisches [1884] p. 103; Suchier, Kleine Beiträge zur Rom. Sprachgeschichte, (Miscellanea Linguistica in onore di Graziado Ascoli, 1901, p. 72). Il est probable que G. Paris qui a longtemps soutenu l'origine anglo-saxonne du mot lai se serait rangé à cette opinion. Cf. Poèm. et lég. du moyen-âge, [1900], p. 143, note I "Je dois dire que cette étymologie [de l'anglo-saxon], que j'ai crue autrefois presque certaine, me semble maintenant sujette à caution." Voir aussi Rom., XXX, p. 569. 
appliqué à ce rythme original, à cette cadence spéciale qui faisaient le charme des "lais bretons" et les distinguaient dès l'abord des airs proprement français. Dès 1159, dans le fabliau de Richeut nous rencontrons déjà le lai breton (chanson) comme une chose parfaitement connue. Puis le mot a fini par s'appliquer à certaine disposition rythmique des chansons françaises qui reproduisait peutêtre l'allure des anciens airs bretons: ${ }^{1}$ nous avons là le lai lyrique du XIII e siècle, dont le lai du Chevrefeuil nous offre un gracieux spécimen. ${ }^{2} \mathrm{Au}$ XIV e siècle nous retrouvons le lai; il a retenu les caractéristiques qu'il avait au siècle précédent: il se distingue toujours par une dissemblance voulue dans la structure des couplets et la disposition de leurs rimes; mais nous sommes à un moment où les anciennes formes lyriques si variées, si malléables, si personnelles font place à des formes nouvelles dont l'arrangement est fixé une fois pour toutes. Le lai n'échappe pas à cette tendance. Eustache Deschamps ${ }^{3}$ en formule les lois et le lai prend désormais place à côté de la ballade et du rondeau parmi les genres à forme fixe. Autre changement important: il ne se chante plus, il se récite ou se lit.4 Du vocabulaire de la technique musicale le mot a décidément passé à celui de la technique poétique. Mais ce qui est au fond de toutes ces significations, du reste très voisines, c'est l'idée générale de mélodie, de cadence, de rythme. Le mot lai qui, on vient de le voir, appartenait aussi bien au vocabulaire courant 5 qu'au lexique spécial des musiciens, puis des poètes lyriques a survécu dans un de ses emplois jusqu'au XVI e siècle. Il a disparu avec les anciens genres à forme fixe, dont il avait fini par désigner surtout une des variétés. Quant au sens de poème narratif, il est inconnu, nous l'avons vu, avant Marie: c'est elle qui l'a introduit dans la langue, sans dessein bien arrêté, par suite d'une

1 C'est l'opinion de M. Suchier, Gesch. der fr. Litt., p. I20. C'est aussi celle de M. Jeanroy (Lais et Descorts frangais, p. XV): il cite ì l'appui deux vers d'un lai français dont le témoignage me semble comme à lui emporter la conviction: "El lai des Hermins / Ai mis reson roumance." Ce texte, ajoute M. Jeanroy, „oppose au 'lai' lui-méme, c'-à-d. à la mélodie que l'auteur a trouvée toute faite, la 'raison romane', c'-d-d. les paroles françaises qu'il y a adaptées." - L'introduction du lai de Doon, que nous avons citée p. 47, si elle ne prouve rien d'autre, prouve du moins que, dans l'opinion de l'auteur, il courait des mélodies celtiques sans paroles, toutes prêtes par conséquent à s'adapter à un texte français.

2 On a la collection complète de ces lais dans Lais et Descorts français $d u$ XIIIe siècle, texte et musique, publiés par Jeanroy, Brandin et Aubry. Paris, 1901.

3 L'art de dictier (Euvres complètes d'Eustache Deschamps, publiées par G. Raynaud, t. VII, p. 287 sqq.). Cf. t. XI, p. 131 sqq.

- Ceci s'applique en tout cas a Deschamps. Voir Art de dictier, t. VII, p. 270-27I et la note de M. Raynaud, t. XI, p. 156, n. I.

5 Lai au sens général de chanson est encore attesté au XVe siècle. Voir par ex. Villon, Grant Testament, v. 973. (Ed. Longnon, p. 61.): „Item, d maistre Ythier Marchant | Donne, - mais qu'il le mette en chant - | Ce lay, contenant des vers dix." Suit, quelques vers plus loin, le lay, qui est une espèce de rondeau. 
confusion qui devait nécessairement se produire entre le lai breton auquel elle se référait sans cesse et le conte français qui prétendait retracer l'origine de ce lai. Le terme, quoique peu justifiable en soi, était commode pour désigner cette variété nouvelle du conte en vers que créait Marie: la nouvelle sentimentale sur un sujet se rapportant à la matière de Bretagne. Elle le retint, il fut retenu après elle. Mais l'emploi en fut non seulement très limité, mais toujours assez hésitant. Evidemment, à s'en tenir au texte de Narie, il y avait pour les lecteurs d'alors comme pour ceux d'après de quoi douter: ne faisait-elle pas le plus souvent une soigneuse distinction entre son conte à elle et le lai breton? et à côté de cela n'y avait-il pas quelques cas assez nets où elle appelait son poème un lai? Plus d'un lecteur dut être assez perplexe. L'auteur de Tyolet et celui du Lecheor, après avoir distingué dans leur prologue ou dans le corps de leur récit entre le lai breton et leur conte, finissaient dans le dernier vers par appeler leur production un lai.1 "De Tyolet le lai ci fine" v. 704. "Selonc le conte que j'oi | Vos ai le lai einsint fini." Lecheor, v. I23-4. (Notez le 'selonc'.) D'autres au contraire, et quelques uns parmi les plus anciens, maintenaient rigoureusement la distinction établie par Marie dans la plupart de ses lais: ni Graelent, Désiré et Havelok, ni Tydorel, Doon, le T'rot et le Cort Mantel ne sont qualifiés de lais par leurs auteurs. Tant que nous restons sur le domaine de la matière de Bretagne, c'est à dire tant que par delà le poème français on nous fait entrevoir, avec plus ou moins de conviction, un lai breton, ce scrupule que nous venons de signaler se maintient: évidemment ici c'est encore la tradition de Marie qui s'impose: on est aussi affirmatif qu'elle, mais pas plus. D'autres conteurs, et ceux là ne puisent généralement à aucune source celtique prétendue ou réelle, vont plus loin: ils remarquent la dignité de ton, la noblesse d'allure des lais de Marie, et sans se préoccuper du reste ils retiendront le terme pour en désigner des contes qu'ils ne se soucient pas de qualifier du nom parfois assez peu honorable de fabliau. C'est la dernière transformation de notre mot lai qui désignera ici un fabliau ou conte d'un genre relevé. ${ }^{2}$ Mais ce sens, comme le précédent du reste, est a peine entré dans la langue: il ne semble pas qu'il ait trouvé grande faveur et on a dû l'abandonner assez vite: nous ne le rencontrons guère que dans l'Ombre, le Conseil, le Lay d'Amours et le Vair Palefroy. Quelques copistes donnent aussi le nom de lais à des poèmes comme Narcisse, l'Oiselet, Aristote, Auberée, mais il est à remarquer qu'aucun passage du texte même de ces contes ne les y autorisait. En somme, dans la double acception conventionnelle et factice de conte de Bretagne et de fabliau relevé,

1 C'est aussi le cas de l'auteur de l'Espine, qui fidèle à son habitude continue à tout brouiller et à ne rien comprendre. Il est difficile de démêler si l'auteur de Nabaret appelle son fabliau un lai, ou s'il entend par là quelque chose de différent de son œuvre propre.

${ }^{2}$ Cf. Bédier, Les Fabliaux', p. 10-II, et Hertz, Spielm.2. p. 57. 
le mot lai introduit dans la langue, d'une façon hésitante, dans la $2^{\text {ème }}$ moitié du XII ${ }^{e}$ siècle, n'a vécu, pendant près d'un siècle, que d'une vie atténuée et incertaine, et a fini par périr obscurément d'inanition à la fin du XIII ${ }^{e}$ siècle, ou peut-être dans le ${ }^{\text {er }}$ quart du XIVe siècle. ${ }^{1}$

Il ne semble même pas que le mot pris dans ce sens particulier ait jamais pénétré en provençal. Qu'on examine les nombreux exemples du mot rassemblés par Bartsch dans un article de la Zeitschrift für romanische Philologie:2 lais y est employé souvent pour désigner le chant des oiseaux, et sert fréquemment aussi à nommer une pièce lyrique analogue au descort provençal. Mais nulle trace d'un emploi au sens de poème narratif. Du reste l'origine celtique du mot ou des mélodies et des chansons qu'il désigne y est décelée par l'addition des mots "de Bretagne" ou la mention de sujets clairement empruntés aux Celtes. Ainsi en Provence comme en France le mot - d'origine celtique appartient d'abord au vocabulaire général de la langue courante et désigne un air, une mélodie, une chanson, puis au lexique spécial des poètes lyriques où il s'applique à une forme rythmique particulière. Mais l'influence de Marie ne s'est pas fait sentir si loin et le provençal n'a pas l'air d'avoir adopté le néologisme qu'elle avait réussi à imposer pendant un temps au français.

$\mathrm{Si}$ nous regardons au delà de la France nous obtenons une nouvelle confirmation des résultats auxquels nous sommes arrivés. Le correspondant allemand de notre mot lai, c'est au moyen-âge le terme Leich: or leich signifie toujours air, chanson, mélodie chantée ou exécutée sur un instrument. ${ }^{3}$ Les Allemands n'ont donc pas connu l'autre sens. Il en va un peu différemment des Anglais. Ils nous ont emprunté, probablement au XIII ${ }^{\mathbf{e}}$ siècle - on n'en trouve pas trace auparavant - le mot lai dans toutes ses acceptions, aussi bien dans celle de poème narratif que dans celle de mélodie et de rythme lyrique spécial. Mais la raison en est bien simple: ils ont emprunté la chose avec le mot. Marie avait composé ses

1 Au XIV e siècle, Jean de Condé nous offre en effet quelques exemples de lai où le mot est passé du sens de fabliau relevé à celui de poème didactique en général, de "dit". Voir $L i$ dis des Estas duu monde, v. 4-5 (Ed. Scheler, t. II): „Ce me fait celer dis et lais | Et biaus exemples et biaus contes." Mais il est à noter que des 75 pièces qu'il nous a laisscics, 3 seulement sont appelées par lui lais (le Blanc Chevalier, le Lévrier, l'Ourse). Cf. Lus et Bechés, v. I5 (Scheler, t. III): „Dirai examples, dis et contes | Par devant princes, ducs et contes." (Le lai n'est pas mentionné). Il est probable qu'il ne faut voir dans cet emploi assez rare du mot qu'un archaïsme, un reste attardé du vocabulaire technique de la précédente génération de poètes. Jean de Condé devait tenir le mot de son père Beaudouin dont la vie appartient au XIII e siècle. Cf. li contes des Hiraus de ce dernier, v. 356-358 (Scheler, t. 1): ,[Vilain] onques | N'ama preudome, clerc ne lai | ne chant ne son ne dit ne lai." Le même Baudouin appelle par trois fois lai (v. 1624, 2830, 3062) li prisons d'amours qu'il désigne ailleurs sous le nom plus approprié de "traitié" (v. 98).

I, p. 58.

- Hertz, Spielm. ${ }^{2}$, p. 343 . 
lais en Angleterre et ils y étaient restés populaires: nous avons une traduction anglaise de Fraisne, et plusieurs versions ou remaniements d'une traduction, de Lanvial. Nous avons aussi un lai d'orphée qui n'est certainement qu'une traduction d'un poème français aujourd'hui perdu. Il était donc naturel que ce sens si spécial de lai $=$ poème narratif entrât en Angleterre, avec les compositions qu'il servait ou pouvait servir à désigner en France. Cependant là encore il ne s'est pas fait accepter sans réserve. Le long prologue du lai d'Orphée nous raconte avec de copieux détails tirés en grande partie de Marie de France ce qu'étaient les lais chez les Bretons, pourquoi et comment on les composait: mais l'auleur ajoute simplement qu'il va nous raconter une de ces aventures qui donnaient naissance aux lais, et nulle part il n'appelle son poème un lai. Orphée est de la fin du XIII siècle. Au XIV ${ }^{\mathrm{e}}$ siècle, c'est à dire à un moment où le mot lai signifiant poème narratif est en France tombé en désuétude complète, les Anglais continuent à nous parler des lais bretons: mais on ne fait plus bien la distinction entre les lais bretons, que personne à ce moment n'a jamais lus ou entendus et les contes français, qualifiés lais, qui en sont les uniques représentants. On voit bien que ces derniers peuvent difficilement se chanter et d'autre part on sait que les lais bretons dont il est question dans les prologues de Marie étaient essentiellement des chansons. De là une certaine perplexité, de là le passage si connu de Chaucer où il s'arrange d'une façon charmante à concilier toutes les opinions et à satisfaire tout le monde:

Thise olde gentil Britons in hir dayes

Of diverse aventures maden layes,

Rymeyed in hir firste Briton tonge;

Which layes with hir instrumente they songe,

Or elles redden hem for hir plesaunce.

And oon of hem have $I$ in remembraunce,

Which I shal seyn with good wil as I can. ${ }^{1}$

A la fin du XIV et au commencement du XV ${ }^{\text {e }}$ siècles les auteurs d'Emare, The Earl of Tioulouse et Sir Gowther se réfèrent encore à des lais de Bretagne: entendez par là des poèmes français que leurs auteurs ou des copistes avaient qualifiés, à plus ou moins juste titre, de lais. ${ }^{2}$ Ainsi le mot lai (plus tard lay), signifiant

1 Canterbury Tales, v. $11021-11027$ (The Prologue of the Frankeleyns Tale). M. Rajna (Rom., XXXII, p. 239) soupçonne que ce prologue est inspiré de celui d'Equitan. Chaucer cherche évidemment à reproduire ici l'introduction qui était de style dans les lais bretons Son conte du Franklin n'a du reste rien à voir avec la matière de Bretagne: c'est ce que M. Rajna a mis hors de doute dans l'article que je viens de citer. Une preuve de plus que les prologues où on nous annonce un conte celtique ne sont pas toujours veridiques.

2 Voir G. Paris, Le Roman du Comte de Toulouse, Paris-Toulouse, 1900, p. 7, note 2. Emare et The Earl of Toulouse doivent provenir de 
conte, a eu un développement parallèle à celui du français lai pris dans le même sens: seulement il apparaît un siècle après, comme il est naturel, et il se prolonge aussi près de deux siècles plus tard. 1

20. Quel rapport y a-t-il entre les lais 'épiques' de Marie et les lais lyriques bretons? Seulement un rapport assez vague d'origine. Les contes de Marie remontent à des récits antérieurs (ce qu'elle appelle l'aventure') et ces mêmes récits ont donné naissance à des lais bretons. Reprenons encore une fois l'examen de ses prologues: jadis, au temps des anciens Bretons, ${ }^{2}$ quand une aventure était arrivée parmi les barons, ${ }^{3}$ on prenait plaisir à se la raconter, elle circulait de bouche en bouche; 4 puis c'était une mode très en honneur que d'en faire un lai pour en conserver le souvenir; 5 ce lai portait généralement le nom du héros de l'aventure; 6 c'était une mélodie qu'on exécutait sur la harpe ou la rote et qu'on pouvait probablement aussi chanter sur des paroles; ${ }^{7}$ parfois le lai n'était composé que longtemps après que l'aventure elle-même était arrivée: ${ }^{8}$ il pouvait donc $y$ avoir des aventures qui se transmettaient sans donner naissance $\grave{a}$ un lai. Voilà ce que nous apprend Marie. Il y a donc deux séries parallèles et correspondantes: d'une part des contes nés du récit de l'aventure, d'autre part des lais composés à l'occasion de ces récits. Mais faisons attention que pour nous ces deux séries sont fort inégalement représentées: nous avons bien les contes de Marie, sous une forme tangible et qui représente une version définie de récits déjà anciens; mais en ce qui concerne les "lais" nous sommes moins bien partagés: non seulement en admettant que des chansons accompagnassent la mélodie, nous n'avons aucune de ces chansons, mais leur existence même n'est attestée que dans un cas: Marie nous dit expressément que la note du lai de Guigemar, qu'on dit en harpe et en rote, est "bone à oïr", d'où nous pouvons conclure qu'elle a entendu exécuter ce lai. Là nous tenons les termes correspondants des deux séries parallèles, mais nous ne les tenons que là. Partout ailleurs nous n'avons qu'un

pcèmes français assez étendus. Quant à Sir Gowther, il est possible que le lai français auquel il se réfère soit Tydorel.

1 Le mot lay existe du reste encore en anglais moderne, avec un sens, il est vrai, assez flottant: tantôt il est synonyme de ballud ct désigne alors „a narrative poem, especially one in simple style and light meter ,, tantôt c'est l'idée de chant, de lyrisme qui domine: dans son Dictionnaire étymologique Skeat, s. v. lay, ne donne comme définition que "a song, lyric". C'est en somme toute l'histoire complexe du mot qui se reflète dans sa présente acception.

2 Equit., v. 3; Elid., v. 1182.

3 Equit., v. 1-3.

- Equit., v. 5-6; Prolog., v. 36; Laustic, v. 157 etc.

s Prol., v. 35-38; Equit., v. 7-8; Elid., $1183-4$; Guing., v. 675-6.

- Chaitiv., v. 201-230; Elid., v. 21-26; Guing, v. 677.

7 Guig., v. 885-86.

8 Yonec, v. 559-560. 
nom, sans la moindre allusion à une composition musicale quelconque. Et souvent ce nom est amené de façon telle qu'il nous donne l'impression d'être inconnu du public de Marie. Il est remarquable que nous ne trouvions nulle part, chez aucun auteur, une allusion à une forme musicale de ces lais bretons cités par Marie. Il n'y a qu'une exception: le lai lyrique du Chèvrefeuil est mentionné plusieurs fois; mais là les paroles même de Marie (v. I I6) nous donnent à penser qu'il y avait une chanson française du Chèvrefeuil, à laquelle peuvent se rapporter toutes les citations, ${ }^{1}$ y compris celle qu'elle fait elle-même d'un lai du Chèvrefeuil.

En résumé, il y a eu au XII ${ }^{\circ}$ siècle des mélodies bretonnes fort goûtées de nos ancêtres; mais elles ont disparu sans laisser de trace dans la littérature en dehors d'une multitude de mentions élogieuses, mais vagues. ${ }^{2}$ Nous pourrons essayer de nous représenter ce qu'elles étaient, mais ce sera à l'aide d'hypothèses qui, toutes plausibles qu'elles seront, resteront des hypothèses. En ce qui nous concerne il n'y a que demi-mal: Marie a certainement voulu faire profiter son recueil de la vogue des lais bretons, mais ce n'est pas d'eux qu'elle tient ses contes. Nous n'avons aucune raison de croire que des chants de harpe aient jamais, à aucun moment, formé un anneau quelconque de la chaîne qui relie l'aventure bretonne au lai français, et en tout cas dans la transmission des légendes celtiques aux Français on ne voit pas que les lais musicaux aient joué d'autre rôle que de fournir cette appellation de 'lai' à laquelle du resté était réservée (de nos jours plus encore qu'au moyen-âge) une si brillante fortune.

Les lais de Marie de France sont des contes qui étaient déjà des contes - et probablement l'avaient toujours été - dans la tradition à laquelle elle les empruntait. Quelle était la nature de cette tradition? On admet volontiers d'ordinaire que Marie n'a fait que recueillir des contes oraux. Cependant elle nous dit nettement le contraire au début de Guigemar:

1 M. Suchier réfère la mention du „sonet del Chevrefoil“ faite par la „Geste du Loherains" (cité par P. Paris, Rom. de la Table $R$. 1, p. 13) au lai lyrique du Chèvrefeuil. (Gesch. der fr. Lit., p. 120.) Ce lai ne date probablement que de la fin du XII e siècle, mais il pouvait y avoir une chanson française du même nom, plus ancienne.

2 Un passage de la chanson de Horn (Ed. Brede et Stengel, v. 2830 -2844) très souvent cité et discuté (voir Birch-Hirschfeld, dans Ersch und Grubers Allg. Encykl., s. v. lais; Hertz, Spielm.2, p. 45; Jeanroy, dans Litt. $f r$. de Petit de Fulleville, I, p. 398) est moins précis qu'il ne le semble au premier abord. Les mols si cum funt cil Bretun de tel fait custumer (v. 2841) ne se rapportent vraiment qu'aux vers 2839 et 2840 . De sorte que tout ce que nous apprenons sur les harpeurs bretons se ramène à ceci, que comme eux Gudmod notait son lai haut et clair. Cela ne nous avance pas beaucoup. Tous les détails qui nous sont donnés dans les vers qui précèdent et dans ceux qui suivent (prélude, ritournelle, etc.) ne s'appliquent sans doute pas plus aux harpeurs bretons qu'aux jongleurs français du XII e siècle en général. 
El chief de cest comencement

sulunc la letre et l'escriture

vos mosterrai une aventure ... (v. 22-24.)

Dans une note de la Romania, ${ }^{1}$ Gaston Paris explique qu'il faut entendre: "Je vous montrerai par écrit, etc." et cette interprétation a été acceptée par tous les critiques qui se sont occupés des lais. ${ }^{2}$ Elle est pourtant très contestable. Notons d'abord que montrer, ${ }^{3}$ sans autre détermination, est courant chez Marie au sens de conter; remarquons ensuite que quand elle veut dire 'montrer par écrit'elle le dit nettement:

Pur ceo nus mustre par escrit, meinte feiz est trové et dit.

(Fables, 83, v. 43-44.)

Et puis considérons la façon dont elle commence la plupart de ses fables qui sont, nous le savons, traduites d'un original anglais: les formules peuvent être différentes, mais Marie s'y réfère toujours à son modèle écrit. Ainsi: D'un leïn cunte li escriz. I 4 , v. I ; Par vieil essample en escrit truis. 17, v. I. Or la fable 3 commence ainsi:

Sulunc la letre des escriz

vus musterrai d'une suriz. (v. I-2.)

Il n'y a pas de raison sérieuse de croire que Marie commençant l'une de ses fables et l'un de ses lais par la même formule lui attribue un sens différent dans les deux cas. Or le sens n'est pas douteux dans le passage des Fables. Il faut donc s'en tenir à l'interprétation la plus naturelle, qui est déjà du reste celle que F. Wolf avait assignée à ce passage. ${ }^{4}$ Ainsi Marie tient son conte de Guigemar d'une source écrite: voilà qui nous éloigne singulièrement des lais musicaux bretons. C'est encore une nouvelle confirmation de résultats auxquels nous étions arrivés par une autre voie. - Ailleurs encore Marie se réfère à un original écrit: c'est dans le Chèrefeuil où, après nous avoir dit qu'elle va nous 'conter la vérité d'un lai' elle ajoute:

5 Plusur le m'unt cunté e dit

et jeo l'ai trové en escrit

De Tristram et de la reïne...

Eilhart d'Oberg nous raconte en effet une aventure analogue à celle que nous rapporte ici Marie ${ }^{5}$ et il y a tout lieu de croire que celle-ci avait trouvé, comme elle le dit, dans une des branches

1 XIV, p. 605, note I.

2 Voir notamment Warnke, Die anonymen Lais, p. 9; Gröber, Grundr., II, I. p. 594 .

${ }^{8}$ Cf. Elid., v. 243-6: Cil ourent enveié avant | un esquiër esperunant, li l'aventure li mustra | et del soldeier li cunta.

- Ueber die Lais, p. 62.

s. 6527 sqq; 7620 sqq. 
du roman de Tristan, les éléments de son gracieux récit. - Nous ne trouvons malheureusement pas dans les autres lais, ni dans un sens ni dans l'autre, de textes aussi nets que les deux que nous venons d'examiner. Ceux qui paraissent les plus clairs à première vue sont très souvent susceptibles de deux interprétations. Le passage suivant de Milun paraît assez probant au premier abord:

\section{De lur amur}

firent un lai li anciën

e jeo ki l'ai mis en escrit

el recunter mult me delit.

Evidemment, dira-t-on, il s'agit là d'une tradition orale recueillie par Marie et mise par écrit pour la première fois. C'est possible, Mais cela pourrait tout aussi bien vouloir dire simplement „Moi qui l'ai écrit [en français, en vers, pour vous, - en tout cas, sous la nouvelle forme qu'il a] j'ai pris beaucoup de plaisir à le conter." Voyez le début du Purgatoire de Saint Patrice:
Al nun de Deu, qui od nus seit,
e qui sa grace nus enveit,
voeil en Romanz mettre en escrit,
Si cum li livre les nus dit ${ }^{1}$. .

Evidemment ici metlre en escrit ne veut pas dire autre chose qu'ecrire. - Plus d'un passage est aussi vague, aussi peu décisif que celui de Milun. Pourtant on a bien l'impression que dans plusieurs cas tout au moins Marie a puisé dans la tradition orale. En somme nous ne serons pas trop loin de la vérité si nous concluons que parmi les contes de Marie les uns lui ont été racontés de vive voix et ont été ainsi fixés par écrit pour la première fois, tandis que les autres existaient déjà sous forme écrite et ont été simplement remaniés ou transformés par elle.

En quelle langue étaient composés ces contes déjd écrits, et en quelle langue lui racontait-on les autres? Que Marie connût le breton - à quelque dialecte celtique que s'appliquât ce terme assez vague - et qu'elle traduisît directement ses lais d'un original breton, on n'en douta pas pendant longtemps et cela semblait assez évident pour ne demander aucune démonstration. C'était au moins l'opinion de G. Paris en 1878.2 Quelques années plus tard cependant, le même savant était amené à exprimer des vues assez différentes. ${ }^{3}$ Selon lui, il eût à peine été possible à Marie, vivant à l'époque où elle vivait, de savoir outre le français trois langues étrangères (latin, anglais, gallois): ce serait lui supposer une instruction extraordinaire. Si l'on remarque de plus comment elle fond, sans s'en douter, en un seul mot (laustic) l'article français

1 Ed. Jenkins, Philadelphia, 1894 .

Roin., VII, p. I.

S Rom., XIV, p. 604 sqq. [1885]. 
le et le gallois aostic, il sera tout naturel d'en inférer qu'elle ne savait pas le breton. Quelques mots anglais et normans disséminés çà et là dans ses Lais nous portent au contraire d̀ croire qu'elle n'a connu les originaux bretons que par l'intermédiaire de traductions françaises et anglaises. Il est curieux que G. Paris se montra beaucoup moins affirmatif par la suite dans son Manuel:1 il se contente d'y dire que Marie avait appris le breton ou au moins l'anglais. M. Warnke accorde ${ }^{2}$ que le mot laustic est en lui-même suffisante preuve que Marie ne savait pas le breton; cependant il refuse ${ }^{3}$ d'admettre avec Paris qu'elle a pu ne connaître les lais bretons que par des récits anglais ou français, et renvoie au v. 886 de Guigemar. "Bone en est a oir la note." Mais quelque sens qu'on donne à ce vers, Marie ne nous y dit certainement pas qu'elle a entendu une chanson bretonne de paroles. Finalement dans un récent compte-rendu 4 de l'édition Warnke, M. G. Cohn a montré qu'on ne saurait tirer aucun argument du mot laustic qui peut n'être après tout qu'une bévue d'un copiste: Marie a certainement pu écrire l'aüstic. - De ces hésitations et de ces contradictions il faut simplement conclure qu'on ne peut pas démontrer, de façon positive, que Marie savait ou ne savait pas le breton. Il reste que nous n'avons aucune raison de croire qu'elle l'ait su et que le contraire, comme l'indiquait G. Paris, serait bien extraordinaire. 5

Le breton écarté, il ne reste comme possible que l'anglais ou le français. Dans laquelle de ces deux langues Marie a-t-elle reçu ses contes? Les quelques mots anglais qui s'y trouvent - gotelef, nightegale - ne prouvent rien en faveur de l'anglais. Car à supposer que l'original de Marie lût anglais, pourquoi ne pas traduire le titre comme l'histoire elle-même? Quel intérêt y avait-il à conserver cette oiseuse relique? On ne peut en pareil cas, si l'on se rappelle où Marie écrivait, invoquer le charme de l'exotisme. Il est impossible de tirer de la présence de ces deux mots un argument sérieux. ${ }^{6} \mathrm{Y}$ a-t-il d'autres raisons plus valables d'affirmer que les sources où Marie puisait étaient anglaises? Il

1 zème éd., p. 90.91 [1890].

2 Lais, p. XXIX-XXX.

8 Lais, p. XXX, note 2 .

- Zts. f. fr. Spr. u. Lit., XXIV, p. 32-35 [1902].

- Marie prend un plaisir évident à conserver des mots bretons dans son texte. Son cas semble étre un peu celui des gens qui ne trouvent tant de charme à des mots ou à des sons étrangers que parce qu'ils ignorent la langue à laquelle appartiennent ces mots et ces sons. Qu'elle garde Guigemar, Guildeluec, Mériaduc, rien de mieux, ce sont des noms propres. Mais pourquoi s'obstiner à appeler un rossignol un laustic? N'y a-t.il pas là comme une recherche d'exotisme qui a dâ séduire le public de Marie, et elle-même tout d'abord? S'il en est ainsi, croira-t-on que Marie ait de gaîté de cœur rejeté les équivalents bretons de frêsne et de chèvrefeuille? N'est-il pas plus probable que sa source - anglaise ou française - qui ailleurs avait retenu le titre breton du lai ne lui fournissait ici qu'une traduction?

- Voir Modern Language Notes, avril 1905. (English words in the Lais of Marie de France.) 
semble bien que non. Quand Marie traduit elle le dit. Voyez l'épilogue de ses $F_{a b l e s,}{ }^{1}$ voyez le début ${ }^{2}$ et la fin $^{3}$ du Purgatoire de St. Patrice. Dans un cas elle traduit de l'anglais, dans l'autre du latin: mais c'est elle qui nous l'apprend, et elle n'en semble pas médiocrement fière. Evidemment elle n'est pas fâchée de faire un peu parade de sa science. Croit-on que si elle eût également emprunté les lais à l'anglais, elle eût négligé de nous le dire? Quand on lit son Prologue-Epilogue on a bien l'impression que c'est quelque chose de tout différent d'une traduction qu'elle va entreprendre. Elle nous dit constamment qu'elle va nous raconter les aventures qu'on lui a rapportées ou qu'elle a lues quelque part. Comment ne pas conclure que ces aventures étaient déjà écrites ou contées en français? C'était certainement la langue qui se parlait autour d'elle:4 elle n'avait donc pas besoin de s'expliquer davantage sur ce point, qui était évident pour ses auditeurs. ${ }^{5}$

La conclusion de toute cette étude, c'est que l'histoire des lais français commence et s'arrête à Marie, et qu'il faut reculer les originaux celtiques de ses lais dans un passé encore plus lointain qu'on n'était porté à le faire. Nulle part elle n'a été en con-

1 „Pur l'amur le cunte Willalme ... | m'entremis de cest livre faire / e de l'Engleis en Romanz traire" v. 9, I 1-12. "Li reis Alvrez, ki mult l'ama,| le translata puis en Engleis, | e jeo l'ai rimé en Franceis | si cum jol truvai, proprement "v. $16-19$.

2 Voeil en Romanz mettre en escrit, | Si cum li livre les nus dit ... | Les peines de l'espurgatoire. v. $3-4,6$.

8 Jo, Marie, ai mis en memoire | Le livre de l'Espurgatoire, | En Romanz. ... v. 2297-99.

- Voy . Espurgat., constinuation du passage cité dans la note précédente: "En Romanz, qu'il seit entendables |A laie gent e cuvenables" v. $2299-2300$.

5 La curieuse introduction de Bisclavret nous apporte peut-être une preuve de plus que Marie puisait à une source déjà française. Après nous avoir dit qu'elle va nous raconter le lai du Bisclavret, elle nous donne la traduction de ce mot étranger au français: "Bisclavret a nun en Bretan, | Garulf l'apelent li Norman. | Jadis le poeit hum oï | e sovent suleit avenir, | hume plusur Garulf devindrent / e es boscages maisun tindrent " v. 3-8. Supposez le mot Garulf connu des auditeurs de Marie, et l'explication est parfaitement suffisante. Mais n'est-il pas singulier que ce mot-là lui-même qui devait nous éclaircir le sens de bisclavret, elle éprouve le besoin de l'expliquer à son tour: Garulf, ceo est beste salvage; | tant cum il est en cele rage, | humes devure, grant mal fait, | es granz forez converse e vait." v. 9-12. Ce mot n'était donc pas clair par lui-même puisqu'il demandait une définition. Sans doute la croyance qu'il atteste pouvait être peu répandue ou à peine connue parmi les auditeurs de Marie, et le mot lui-même avait pu tomber en désuétude. Mais en tout cas il reste que ce mot était pour le public de Marie tout aussi obscur que bisclavret. Pourquoi donc alors l'aurait-elle introduit dans son texte - elle ne s'en servira plus après cette explication du début - si elle ne le trouvait pas déjà dans sa source? C'est ainsi probablement que le conte qu'elle avait lu ou entendu traduisait le titre breton du lai, et elle a reproduit cette traduction parce qu'après tout c'était le seul équivalent français, mais elle a senti que le mot appelait un commentaire, et elle l'a donné. 
tact même indirect avec eux. Voilà pourquoi ces mots de Breton et de Bretagne sont si vagues sous sa plume. Elle entend certainement sous ces mots la Bretagne et les Bretons d'autrefois; 1 mais ne la pressons pas trop sur ce que cela veut dire: elle serait hors d'état de satisfaire les exigences de notre curiosité. Il est à peine exagéré d'affirmer que ces Bretons dont elle nous parle si souvent sont aussi loin dans le passé pour elle qu'ils le sont pour nous. Ces harpeurs bretons qu'on nous a montrés circulant dans les cours et les châteaux, exécutant leurs lais sur la rote et donnant en mauvais français l'explication des paroles bretonnes, ${ }^{2}$ nulle part nous ne les entrevoyons dans Marie ou ses imitateurs. S'ils ont existé, ils appartiennent à une période précédente. Au temps de Marie, il peut y avoir encore des musiciens bretons exécutant des mélodies bretonnes: il est possible que ce soit par un Breton que Marie ait entendre exécuter la note de Guigemar; mais cela même est assez douteux. En tout cas le conteur breton qui est l'intermédiaire entre la légende celtique originale et la forme française est un personnage depuis longtemps disparu. En réalité les contes bretons ont déjà vécu d'une longue vie et passé à travers plus

1 Voir surtout le début d'Equitan: Mult unt esté noble barun | cil de Bretaigne, li Bretun. | Fadis suleient ... faire les lais. v. I sqq. Cf. Milun, v. 531-2: De lur amur et de lur bien | firent un lai li anciën; Elid., v. I-3: D'un mult anciën lai Bretun | le cunte .... vus dirai; v. I182-3: li anciën Bretun curteis | firent le lai. Cf. Brugger, Zts. f. fr. Spr. u. Lit., XX, p. 79 sqq. Nous avons dans tous ces passages de Marie ce que $M$. Brugger appelle le sens I: Bretagne désigne l'Angleterre d'avant la conquête germanique, et Bretons les habitants de cette Bretagne pendant la même période. (Non que la Bretagne armoricaine soit exclue. Guig., v. 25; Elid., v. 30. L'important est que tout ceci est reporté dans un passé lointain. Il ne s'agit en aucun cas de la Bretagne ou de l'Angleterre contemporaines de Marie.) Cf. F. Lot, Rom., XXVIII, p. 4I sqq. Je suis tout à fait de son avis sur le caractère conventionnel de l'expression "lai breton". Il ne sert de rien de citer avec M. Warnke (Lais, p. IX-X) le début ou la fin de Tydorel pour prouver le contraire. Tydorel est un lai très postérieur à Marie, où il ne faut aller chercher aucun renseignement sur les Bretons. Le v. 678 de Guingamor „Einsi l'apelent li Bretun" peut très bien se référer à une source écrite et désigner un temps très reculé dans le passé.

2 Voir surtout G. Paris, Hist. Litt. de la France, XXX, p. 7-8. Il ne semble pas qu'on ait jamais produit aucun argument de fait en faveur de cette opinion, excepté le curieux passage du Roman de Renart cité par M. Bédier (Rev. des deux Mondes, 1891, p. $85 \mathrm{I}$ ), où l'on voit Renart, déguisé en jongleur breton, parler à Ysengrin un singulier baragouin (Ed. Martin, I, p. 66-67.) Mais il faut noter que Renart a ses raisons pour massacrer ainsi le français: il tremble d'être reconnu par Ysengrin à sa voix (Ib., v. 2335). Il se donne donc comme un étranger qui fait effort pour parler français, ce qui lui permet de changer le ton et l'accent de sa voix. On ne sait pas bien de quelle Bretagne il veut parler quand il se dit breton, mais en tout cas il a parcouru l'Ángleterre (v. 236I) et son prétendu baragouin breton n'est qu'un mélange de français écorché et d'anglais: si goditoët (v. 2394) est douteux, ses premiers mots qui ont échappé à M. Bédier, ne le sont guère: „Godehelpe, fait-il, bel sire!" (v. 235I). Si donc il y a intention satirique dans ce passage - ce dont je doute - c'est moins des harpeurs bretons que des Anglais qu'on veut nous y faire rire. 
d'une transformation avant d'arriver à Marie. ${ }^{1}$ Chemin faisant, ils ont subi des additions et des modifications qui ont introduit dans les légendes celtiques bien des éléments étrangers. Quelques uns ont dû devenir presque méconnaissables: on continuait toutefois à les appeler bretons en vertu d'une obscure et vague tradition qui leur assignait une origine celtique. Cette tradition a pu s'attacher à des contes qui. n'avaient rien de celtique. Du reste il a dû devenir de plus en plus difficile de faire le départ entre les contes d'origine sûrement celtique et d'autres d'origine étrangère. Marie recevant ces contes de la tradition orale ou écrite dans des conditions identiques les a tous englobés sous l'épithète de lais bretons, à l'imitation du premier de ses lais, Guigemar, qui semble sûrement celtique. Il se peut qu'elle soit de très bonne foi quand elle nous dit de telle aventure nullement celtique dans le dessein général ou les détails que les Bretons en firent un lai. Mais nous avons vu ce que vaut son témoignage sur ce point et nous pouvons le récuser: à l'égard des Bretons, elle n'était pas mieux renseignée que nous ne le sommes. Son estampille ne saurait suffire à faire d'une histoire quelconque une légende celtique, c'est à dire empruntée aux Celtes. Il est même possible que Marie ait composé elle-même quelques uns de ces lais, c'est à dire leur ait donné pour la première fois une forme littéraire. Il semble bien qu'elle ait recueilli la légende des Deux Amants dans le pays même où cette légende était née, la Normandie.

Il faudrait donc faire deux catégories: les lais qui sont de source indubitablement celtique, parce qu'ils portent encore la trace de leur origine, et ceux qui n'offrant pas de caractéristiques de ce genre ne nous permettent pas de décider s'ils viennent en dernière analyse des Bretons ou non. Mais ce qu'il faut retenir, c'est que ces distinctions ne sont possibles et à vrai dire n'ont de sens que pour les érudits d'aujourd'hui. Marie n'a fait aucune différence entre ces deux groupes. Tous ses lais, sans distinction, elle les a recueillis au même titre. On peut dire qu'elle est à l'égard de ses sources, comme les auteurs des Lais anonymes sont à l'égard de ses Lais à elle. La différence, et elle est grande, c'est que leurs imitations ne nous offrent qu'un pâle reflet de leur modèle, tandis que Marie - et c'est là son originalité - a réellement donné aux contes qu'elle trouvait dans ses sources une forme littéraire durable, à quelques uns peut-être la première forme littéraire qu'ils eussent encore reçue.

Les contes de Marie n'occupent pas de position privilégiée dans la littérature du moyen-âge, si ce n'est celle qu'ils tiennent de leur mérite littéraire. Chacun d'eux nous présente un problème du même genre que celui qui se pose à propos d'un très grand

1 Cf. G. Paris, Rom., XIV, p. 6o7. „Déjà transmis oralement chez les Bretons, puis suivant toute vraisemblance chez les Anglais et les Normands et de plus en plus altérés à chaque étape nouvelle, ils [ces récits] sont arrivés aux mains de notre poétesse dans un état tout à fait fruste et fragmentaire."

Zeitschr, f. rom. Phil. XXXI. 
nombre de contes et de fabliaux de la même époque. L'historiette de Laustic ou la légende d'Eliduc ont avant de venir se fixer dans les vers de Marie passé par des péripéties analogues à celles qu'ont subies le conte de l'Epervier par exemple ou celui de l'Oiselet avant de se retrouver dans les formes françaises que nous en avons. Seulement dans un cas nous avons le point de départ et plusieurs anneaux de la chaine, si le dernier - la source immédiate où ont puisé les trouvères français - nous manque; dans l'autre au contraire - dans le cas de Marie - même quand la couleur celtique d'un conte est indéniable nous ne voyons pas clairement le point de départ. C'est peut-être qu'ici ou bien nous avons affaire à une tradition qui a été constamment orale et ne s'est cristallisée pour la première fois qu'avec Marie, ou bien les originaux écrits plus grossiers, plus frustes ont disparu, comme il est naturel à ce moment dans les limites d'un même language et d'une même littérature, devant la perfection des copies qu'ils avaient inspirées. En tout cas, si en l'absence de tout texte oriental il y aurait eu une certaine témérité à vouloir rattacher l'Epervier ou l'Oiselet à l'Inde, il serait tout aussi imprudent, sur la seule attestation de formules stéréotypées et vagues, de vouloir toujours invoquer les Bretons dans chaque cas où Marie est en cause. Ici comme ailleurs, en dehors de quelques contes où l'imagination celtique a comme laissé sa marque, il faudra, avant d'affirmer, produire des preuves, en attendant de les avoir trouvées, rester dans le doute.

D'où que viennent ses contes, Marie les a faits siens. Les Lais respirent un parfum discret, atténué, perceptible pourtant à qui veut le recueillir, et qui ne rappelle rien d'autre. Plus d'un a voulu s'approprier ce charme subtil et n'y a pas réussi. C'est qu'il résidait moins dans la légende que dans la façon dont elle était contée. Quand on compare Marie à tous ces pauvres imitateurs que son succès a suscités, on se fait une idée plus juste de son mérite: Lanval et Eliduc ne valent pas seulement par la beauté de la légende, il ne serait pas difficile de montrer que comme contes, ce sont de petits chefs d'œuvre d'un art délicat et très conscient. 1 Pour plus d'une raison, il devient de plus en plus chimérique de vouloir retrouver derrière les lais français la forme et le contenu des originaux bretons. Ne nous en plaignons pas trop: nous avons probablement dans les lais de Marie le meilleur de ces légendes, et il est douteux que, dans la longue série de leurs pérégrinations, elles aient jamais trouvé poète pour les rimer on conteur pour les dire qui fût plus vraiment poète ou conteur plus exquis que Marie de France.

1 Voyez le début de son recueil (Guig., v. I-2): „Ki de bonne matire traite, | mult li peise, se bien n'est faite." Nous avons là la part de la tradition, et la part de l'art qui sait s'approprier la tradition et la marquer d'un caractère personnel. Pour une autre expression de l'intérêt qu'elle prend a la technique de son art, cf. Milun, v. 1-4.

Lucien Foulet. 